\title{
Recent advances and progresses in photonic devices for passive radiative cooling application: a review
}

\author{
Amit Kumar Goyal* and Ajay Kumar \\ Jaypee Institute of Information Technology, Electronics and Communications Department, \\ Noida, Uttar Pradesh, India
}

\begin{abstract}
This review covers the recent progress made in the nanophotonic devices-based daytime passive radiative coolers. The radiative cooling capabilities along with the structural description of various natural species are discussed. The design principle along with key characteristics of the omnidirectional solar reflectors as well as thermal adiators is discussed in detail. Several analogues planner one-dimensional and two-dimensional photonic nanostructures and their current state-of-the-art techniques have been discussed. For each kind of the photonic structure, the novelty, measurement principle, and their respective daytime radiative cooling capability are presented. The reported works and the corresponding results predict the possibility to realize an efficient and commercially viable radiator for passive radiative cooling applications. (C) 2020 Society of Photo-Optical Instrumentation Engineers (SPIE) [DOI: 10.1117/1.JNP.14 $.030901]$
\end{abstract}

Keywords: photonic crystal; radiative cooling; photonics; solar spectrum reflector.

Paper 20063V received May 28, 2020; accepted for publication Jul. 29, 2020; published online Aug. 13, 2020.

\section{Introduction}

Cooling represents a significant sector of energy consumption. ${ }^{1}$ It is the most significant driver of peak electricity demand as well as considerable end use of energy globally. ${ }^{2}$ The global energy consumption can be reduced by adopting a cooling strategy that can cool without any electrical input, which is also known as passive cooling technique. The cooling can be achieved if one is able to reach and maintain a temperature below ambient temperature. Radiative cooling, as a passive radiative cooling scheme, does not require any external active devices, such as fans, air conditioners, or thermoelectric. ${ }^{3-5}$ It works on the principle that the atmosphere is almost transparent between 8 and $13 \mu \mathrm{m}$ (also known as "sky window"). Thus, utilizing outer space as a heat sink by allowing the emission of thermal radiation from surface to the universe. ${ }^{6,7}$

In the past few decades, the radiative cooling strategy has been widely explored. Its significance along with potential application in cooling during nighttime was practically demonstrated. ${ }^{8-10}$ A number of different materials showing the capability of intrinsic infrared (IR) emissions for substantial radiative cooling have also been explored previously. ${ }^{11-13}$ These results well demonstrate the capability of the radiative cooling technique. However, the work was mostly limited for the nighttime radiative cooling only. This is because of the availability of suitable materials that possess good IR emission within the atmospheric window. But the primary cooling power requirement usually occurs during the presence of the sun (daytime as compared to nighttime). ${ }^{14}$ The presence of Sun also affects the radiator's efficiency. Therefore, for daytime cooling, both high emissivity (in sky window) and high reflectivity (of the complete solar spectrum) are required. This approach utilizes the universe as a heat sink to release the heat through the atmospheric window and minimize the absorption of incoming atmospheric radiation. Then the surface temperature can be decreased below the ambient. Therefore, certain natural materials, such as polymeric materials, ${ }^{15,16}$ titanium dioxide,,${ }^{17-19}$ silicon nitrides, ${ }^{20}$ and silicon-monoxide $(\mathrm{SiO}),{ }^{21}$ have potential for limited selectivity. But it is difficult to find out a simple bulk material with high emissivity and high reflectivity in the desirable spectrum range simultaneously. ${ }^{22}$ However, many natural species possess the capability to cool down themselves by exhibiting both the aforementioned properties simultaneously. Inspired from natural species,

*Address all correspondence to Amit Kumar Goyal, E-mail: amitgoyal.ceeri@gmail.com 
a lot of work is also carried out to design nanophotonic devices for daytime radiative cooling applications. This facilitates capacity to simultaneously possess a reflectivity of more than $97 \%$ for complete solar spectrum and strong IR emission within the sky window. ${ }^{23-26}$ The development of nanophotonics structures is certainly an important factor that helps in the current resurrection of passive radiative cooling techniques.

Interestingly, one recent article demonstrated the potential of one-dimensional photonic crystal (1D-PhC) structure that can cool down an entity below the ambient temperature using the same technique to that of Sahara silver ants, i.e., highly reflective and emissive for the desirable spectrum range. ${ }^{27}$ Similarly, another recent report has also been reported for this purpose by mimicking the structural attributes of Morpho butterfly wings and successfully demonstrated the passive radiative cooling strategy. ${ }^{28}$ These emerging nanophotonic devices can offer an efficient cooling strategy with their strictly selective and achievable properties and have triggered significant research interest in this area.

Within this context, this review provides sufficient details regarding design principle, radiative cooling characteristics, and recent progress made toward the development of daytime passive radiative cooling devices. Section 2 presents an overview of biomimetic analogy of radiative cooling and motivation behind developing these devices. Section 3 presents a detail working principle of radiative cooling. The approach for nighttime and daytime applications is identified. This section also discusses the design strategy for omnidirectional reflector (ODR). In Sec. 4, we review the different material systems along with various 1D and 2D nanophotonic devices developed for daytime passive radiative cooling applications in the earlier studies. Their working principles and performances of the reported designs are thoroughly investigated and possible efficiency improvement strategies are discussed. Section 5 discusses the current challenges and scopes of daytime passive radiative cooling technique and finally Sec. 6 concludes the review.

\section{Radiative Cooling's Biomimetic Analogy}

While the proposed passive daytime radiative cooling concept is potentially transformative for metallic structures, the idea of selective passive radiative cooling is not new to nature. Various insects and animals living in hot environment, such as Morpho butterfly, Pompeii worm (Alvinella pompejana), Sahara Desert ant (Cataglyphis bicolor), and water bear or Tardigrade (Hypsibius dujardini) possess the capability to withstand extreme climate changes. For example, Pompeii worm and Sahara Desert ant can withstand a temperature as high as $176^{\circ} \mathrm{F}\left(80^{\circ} \mathrm{C}\right)$ and $122^{\circ} \mathrm{F}\left(50^{\circ} \mathrm{C}\right)$, respectively. ${ }^{29,30}$ Water bear is capable of surviving the temperature more than $302^{\circ} \mathrm{F}\left(150^{\circ} \mathrm{C}\right) .^{31}$ This happens only because of their unique body structure that helps them to overcome the heating challenges. Alongside, beetles (Coleoptera) exhibit a number of interesting optical phenomena, i.e., metallic colors and spectral iridescence. ${ }^{32}$ Recently, the white structure presents on beetles' elytra has also attracted researchers' interest to explore this mechanism. Many authors have further explored the optical functionalities of these species to develop various new biological photonic structures. ${ }^{33-36}$

If an entity keeps on absorbing the significant portion of solar radiation, it will not survive. Therefore, most of the natural substances have their body structures that help them to reflect solar radiation. For example, Morpho butterfly has empty pores within the thin membranes, which helps them to scatter near-IR radiation and avoid excessive heating under sunlight. ${ }^{37}$ The Morpho butterfly image and corresponding pictorial representation of internal structure are shown in Figs. 1(a) and 1(b), respectively. Furthermore, these species also exhibit strong emissive properties in mid-infrared (MIR) range, thereby using universe as heat sink as explained earlier. ${ }^{28,38}$ Similarly, the internal structure of Bistonina biston butterfly's wings also exhibits spectrally selective emissivity values. This helps butterfly to cooldown the wing surface. Because of its unique structure, it exhibits a high emissivity in the MIR (2.5 to $16 \mu \mathrm{m})$ to dissipate heat and it is highly reflective to the solar spectrum, which helps it to cool the wings. ${ }^{39}$

Saharan silver ant is another widely studied insect that possesses the capability to stay cool in the Saharan Desert under the direct sunlight. The amazing temperature management ability of Saharan silver ants is also due to their unique structural geometry. The Saharan ants are covered with a dense array of hairs with triangular shapes on both the top and sides of their bodies as 


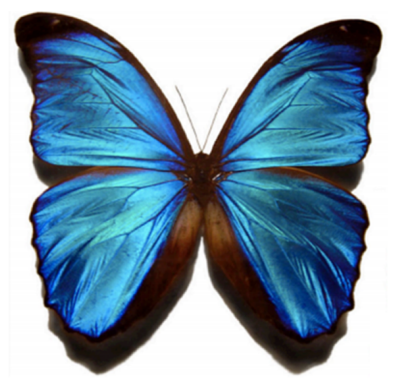

(a)

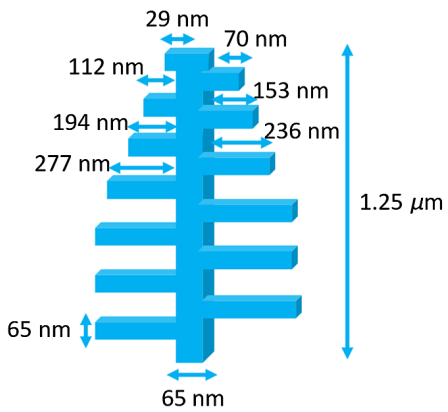

(b)

Fig. 1 (a) The Morpho didius butterfly image (image source: wiki image) and (b) schematic of corresponding internal structure.
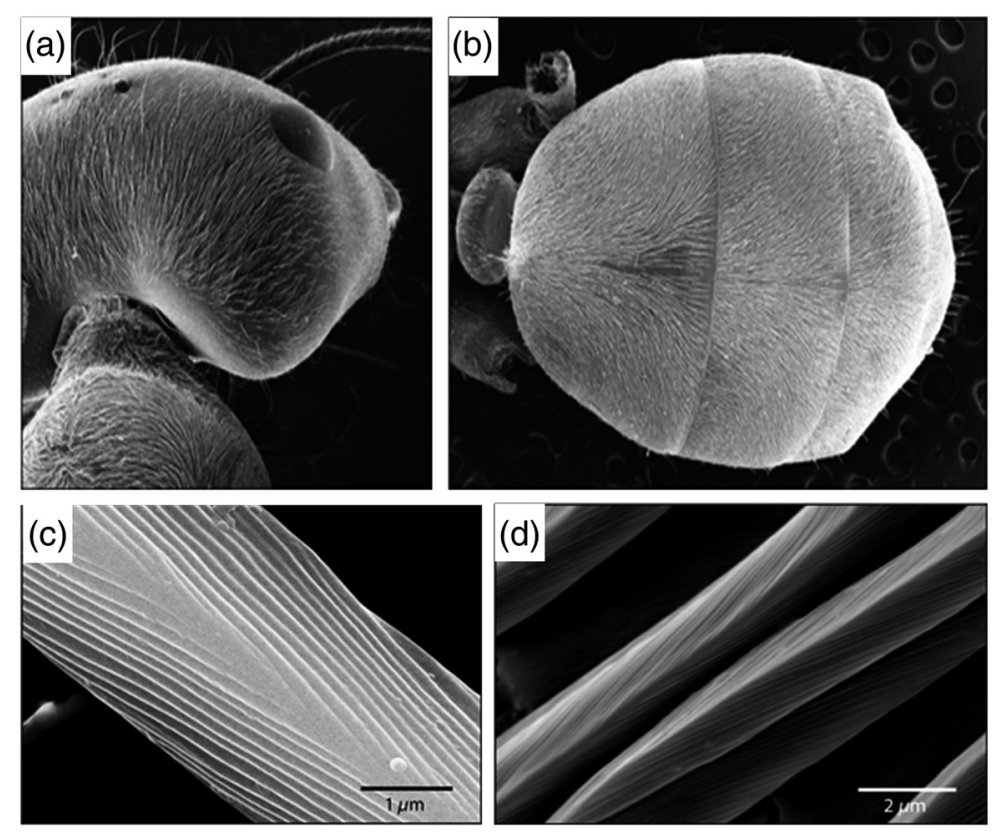

Fig. 2 The Sahara silver ant structure. (a) Scanning electron microscopy (SEM) image of ant's head covered with hairs. (b) SEM image of ant's abdomen covered with hairs. (c) and (d) The close-up look via SEM imaging (Copyright 2016 Willot et al. ${ }^{40}$ ).

shown in Fig. 2. ${ }^{27,40}$ It has been recently demonstrated that the periodical arrangement of their hair structures makes them reflective to the solar spectrum $(0.4$ to $1.7 \mu \mathrm{m})$ and highly emissive in the MIR range ( 2.5 to $16 \mu \mathrm{m}$ ). ${ }^{27}$ These uniquely shaped silvery hairs protect and enable the ants to maintain lower body temperatures by two main mechanisms (i.e., reflection of most of the solar spectrum and transferring heat to the universe by improving the emissivity in the MIR region) similar to the Bistonina biston butterfly. The combined effect helps the silver ant to maintain steady-state body temperature.

Another example present in nature is the cocoon made by wild silk moths. The cocoons comprise a number of fibers arranged randomly and scatter the incident light. This attributes to high solar reflectivity along with high MIR emissivity. ${ }^{41,42}$ This helps moth pupae from overheating under the direct sun. In 2018, Choi et al. ${ }^{43}$ explored the internal structure of silkworm fibers and found the presence of microcavities. These microcavities are responsible for both reflections of the solar spectrum and suppression of the transmission spectrum via Anderson localization.

The thermoregulatory solutions demonstrated by natural species are the examples that show the capability of an animal to control electromagnetic waves over an extremely broad range of 
the spectrum (almost complete solar spectrum). These remarkable effects observed in these natural species could be very interesting and have a massive technological impact and inspiration for the development of nanophotonic devices for passive radiative cooling applications.

\section{Fundamental Principles of Passive Radiative Cooling}

\subsection{Design Principle of Passive Radiative Cooler}

In this section, the fundamental principle behind the passive radiative cooling strategy is presented. The environmental radiations comprise both solar radiation and emitted IR radiation from the Earth's surface, which is further used to maintain the energy balance. ${ }^{44}$ This energy balance is required to maintain an equilibrium temperature. When a surface under consideration is exposed to the environment, the cooling power $P_{\text {cool }}$ is considered to be the resultant out-going radiative energy flux and can be represented by

$$
P_{\text {cool }}(T)=P_{\text {rad }}(T)-P_{\text {atm }}\left(T_{\text {amb }}\right),
$$

where $P_{\text {rad }}(T)$ and $P_{\text {atm }}\left(T_{\text {amb }}\right)$ are the thermal emission of the radiative cooler and thermal atmospheric radiations with the operating temperature $T$ and $T_{\mathrm{amb}}$, respectively. These can be calculated by

$$
\begin{gathered}
P_{\mathrm{rad}}(T)=A \int \mathrm{d} \Omega \cos \theta \int_{0}^{\infty} \mathrm{d} \lambda I_{B}(T, \lambda) \varepsilon(\lambda, \theta), \\
P_{\mathrm{atm}}\left(T_{\mathrm{amb}}\right)=A \int \mathrm{d} \Omega \cos \theta \int_{0}^{\infty} \mathrm{d} \lambda I_{B}\left(T_{\mathrm{amb}}, \lambda\right) \varepsilon(\lambda, \theta) \varepsilon_{\mathrm{atm}}(\lambda, \theta),
\end{gathered}
$$

where $\varepsilon_{\text {atm }}$ represents the spectral and angular atmospheric emittance. The lowest achievable temperature directly depends on the net cooling power. Higher net cooling power leads to attaining the lowest temperature. This also depends on various other factors such as incident solar power and conductive heat transfer during daytime. Thereby, a passive radiative cooler is a structure that can provide temperature lower than that of ambient air. For a surface that is directly exposed to the atmosphere under sunlight (as shown in Fig. 3), the net radiative cooling power can be obtained by modifying Eq. (1) and represented by following equations: ${ }^{45}$

$$
\begin{gathered}
P_{\text {net }}^{\mathrm{rad}}=P_{\mathrm{rad}}-P_{\mathrm{atm}}-P_{\text {solar }}, \\
P_{\text {net }}=P_{\text {rad }}-P_{\text {atm }}-P_{\text {solar }}-P_{\text {nonradiative }},
\end{gathered}
$$

where $P_{\text {rad }}$ is the thermal emission of the radiative cooler (surface), $P_{\text {atm }}$ is the absorbed atmospheric radiation power on the surface, and $P_{\text {solar }}$ is the absorbed incident solar radiation power on the surface. It is noteworthy to mention that Eq. (4) represents the net radiative cooling of a surface by considering the effect of only atmospheric and solar radiations. The structure under

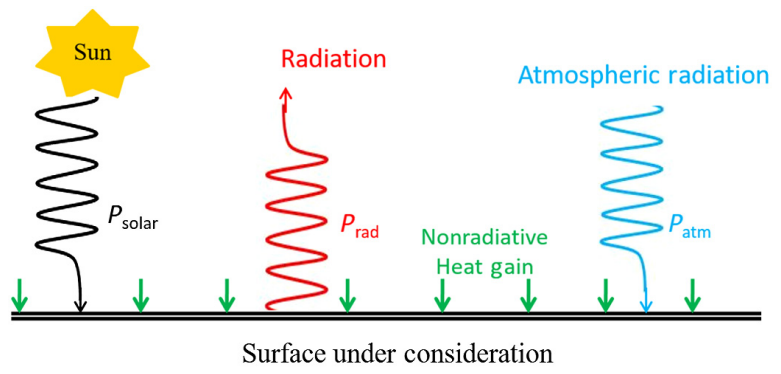

Fig. 3 Schematic structure of the heat transfer on a surface under consideration. 
consideration is directly exposed under the sunlight. To consider other surrounding effects or nonradiative heat exchange process, Eq. (4) can be modified as Eq. (5).

Figure 3 represents the pictorial working principle of daytime passive radiative cooling devices, where the mentioned terminologies are already explained earlier. According to Eq. (5), $P_{\text {solar }}$ and $P_{\text {nonradiative }}$ behave as parasitic cooling losses those attribute to the reduction in the overall cooling efficiency. This further leads to increase in the cooling time to obtain the thermal equilibrium. In addition, the cooling efficiency also depends on the emission characteristic of the radiator, i.e., broadband or selective radiator. The ideal broadband radiator possesses the capability with unity emittance throughout the IR spectrum along with negligible absorption for the complete solar spectrum in daytime radiative cooling applications. Spectrally selective cooler possesses the unity emittance only in the atmospheric window. ${ }^{46}$

The structural net radiative cooling efficiency or cooling time can be improved by minimizing the absorption of atmospheric and solar radiation. Generally, the radiation property of a perfect absorber (blackbody) is also very strong and research is being carried out to design perfect absorber. ${ }^{47,48}$ The solar radiations are considered to be equivalent to that of a blackbody radiations for an effective temperature of around $5800 \mathrm{~K}$. Figure 4(a) represents the normalized atmospheric transmission for different wavelengths, where it appears to be highly transparent from 8 to $13 \mu \mathrm{m}$. One more sky window also appears between 16 and $23 \mu \mathrm{m}$. Due to its weak transmittance, it has very less significance for radiative cooling and neglected in general. The Earth's atmosphere exhibits good emissivity outside this window. Coincidentally, the peak thermal radiation characteristic of a blackbody (at around $300 \mathrm{~K}$ ) overlaps with the atmospheric window, as shown in Fig. 4(a). The emitted IR rays pass through the Earth's atmosphere and move into the outer space. Thereby, it uses universe as a heat sink. Figure 4(b) shows the spectral irradiance graph of AM 1.5 solar spectrum (at irradiance of about $1000 \mathrm{Wm}^{-2}$ ). The absorption of this power can drastically affect the cooling performance of the radiator. Apart from these, atmospheric conditions also affect the performance of daytime radiative cooling structures.

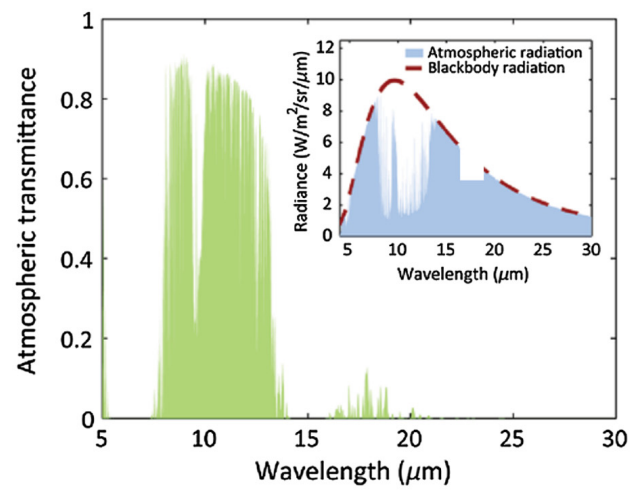

(a)

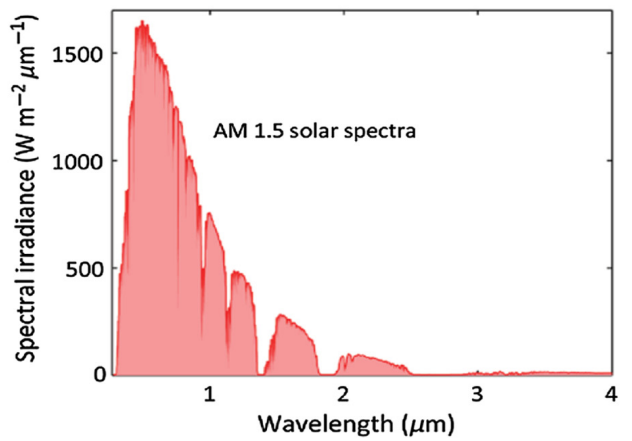

(b)

Fig. 4 (a) The effect of wavelength on atmospheric transmittance and spectral irradiation (reprinted from Zhao et al., ${ }^{6}$ with the permission from AIP) with the comparison of blackbody radiations. (b) The spectral irradiance graph of AM 1.5 solar spectrum (data from Ref. 136). 
This has already been discussed in various earlier reports. ${ }^{49-52}$ To minimize the effects of incoming solar radiation, an IR transparent reflector needs to be integrated on the top side of the surface. Alternatively, a cooling device itself can be designed that possesses the capability to serve as both solar reflector and IR radiator simultaneously.

\subsection{Design Principle of Dielectric Reflectors}

The dielectric reflector is a structure that possesses the capability to reflect the complete solar spectrum at all incident angles. This is also considered as omnidirectional broadband reflector. The structure works on the principle of distributed Bragg reflector (DBR), where optical length optimization is carried out to enhance the reflectivity. Optical length can be varied by changing layer properties, such as height, RI, and porosity. Each layer causes a partial reflection of incident radiation as shown in Fig. 5. In Fig. 5(a), layer "A" corresponds to the material of high RI and layer " $\mathrm{B}$ " is of low RI. The structure is designed in such a way that all the reflected waves constructively interfere to give an overall high reflection. This constructive interference condition can easily be governed by Bragg's law as shown in Fig. 5(b).

The structure can be designed using a quarter-wavelength Bragg reflector. The analysis can be carried out by considering a material system of high and low refractive indices that should also have high emissivity in the atmospheric window. Generally, the structure is designed to reflect a broadband spectrum centered at wavelength $\left(\lambda_{0}\right)$. For normal incident, the layer thicknesses are obtained using Eqs. (6) and (7), while Eq. (8) is used for oblique incident.

$$
\begin{gathered}
n_{H} d_{H}=\frac{m \lambda_{0}}{4}, \\
n_{L} d_{L}=\frac{m \lambda_{0}}{4}, \\
\frac{\lambda_{0}}{2}=d_{L} \sqrt{n_{L}^{2}-\sin \theta^{2}}+d_{H} \sqrt{n_{H}^{2}-\sin \theta^{2}},
\end{gathered}
$$

where $n_{H}$ and $d_{H}$ are the RI and thickness of high refractive index material, respectively, $n_{L}$ and $d_{L}$ are the RI and thickness of low refractive index materials, respectively, $\theta$ is the incident angle, and $\lambda_{0}$ is the center reflected wavelength. The width of reflection spectrum depends on various parameters, such as index contrast between layers and number of bilayers. However, one needs to overcome the trade-off between high reflectivity and high full-width-half-maximum. Theoretically, the structure can be designed and optimized by following various classical optimization methods, such as needle optimization, ${ }^{53,54}$ simulated annealing, ${ }^{55}$ jump method, ${ }^{56}$ and memetic algorithm. ${ }^{57}$

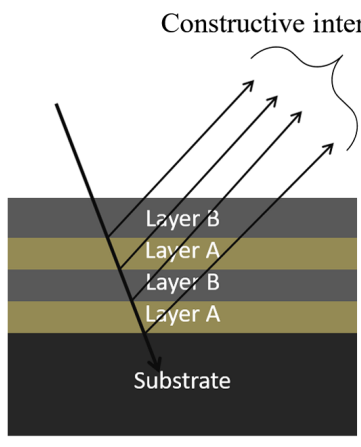

(a)

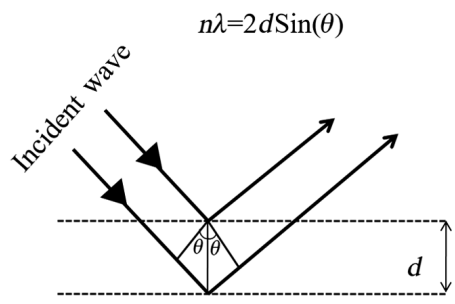

(b)

Fig. 5 Schematic diagram of (a) DBR structure and (b) Bragg's law. 


\section{Recent Progress and Discussions}

Achieving subambient radiative cooling during nighttime is straightforward. But, in the daytime, the presence of the sun creates the difference. The cooling scenario can further be divided into four major parts, i.e., daytime above-ambient and subambient, nighttime above-ambient and subambient as shown in Fig. 6. Most of the radiators are designed to exhibit the characteristic similar to these ideal emissivity/absorptivity spectral curves. For nighttime cooling, 2.5- to $50-\mu \mathrm{m}$ wavelength range is of interest. For daytime, it is of around 0.3 to $50 \mu \mathrm{m}$ (i.e., extra 0.3 to $2.5 \mu \mathrm{m}$ is because of solar spectrum).

Subambient daytime cooling applications require the emissivity equivalent to blackbody emissivity in the atmospheric window and emissivity close to zero in the solar spectrum. This radiator has to be selective as explained in Sec. 3. The major research works for nighttime subambient radiative cooling devices are based on developing structures for selective IR emission in the sky window that is represented by a solid blue line in Fig. $6{ }^{58-61}$ However, for daytime cooling (either above-ambient or subambient) as shown by black solid and red dashed lines in Fig. 6, the major challenge is to achieve both high solar reflectance and high emissivity over the atmospheric window. A lot of research studies have been carried out to design structures that can reflect complete solar spectrum as well as are highly emissive in the atmospheric transparent window. The following section describes various strategies to design a broadband solar reflector followed by their applications in realizing daytime radiative cooling devices.

\subsection{Dielectric Omnidirectional Reflector}

The recent progress on developing nanophotonic devices based on radiators for passive radiative cooling applications has provided a new direction toward achieving highly efficient devices. Thus, improving the ability to effectively work directly under the sun by maintaining their temperatures below the ambient. Photonic crystal (PhC)-based structures are extensively investigated because of their light controlling and bandgap properties. ${ }^{62-67}$ These properties are further used to design various one-dimensional (1D) and two-dimensional (2D) PhC-based structures for passive radiative cooling applications. ${ }^{68-71}$ The PhC structure shows a complete photonic bandgap (PBG), when no electromagnetic mode is allowed to propagate through the structure and behaves as a perfect reflector. ${ }^{72,73}$ The width of reflection spectrum (or PBG) is important while designing an ODR specifically, for applications such as daytime radiative cooling. ${ }^{74}$ To achieve daytime radiative cooling, an ODR is required that should have the capability to reflect complete solar spectrum for both TE and TM polarization. ${ }^{75-77}$ The metallic reflectors are widely accepted for reflection purposes but their widespread uses are limited because of their lossy properties at optical wavelengths. ${ }^{78}$ Therefore, dielectric-based structures are considered to be good alternatives for this purpose as they also provide design flexibility in terms of reflection

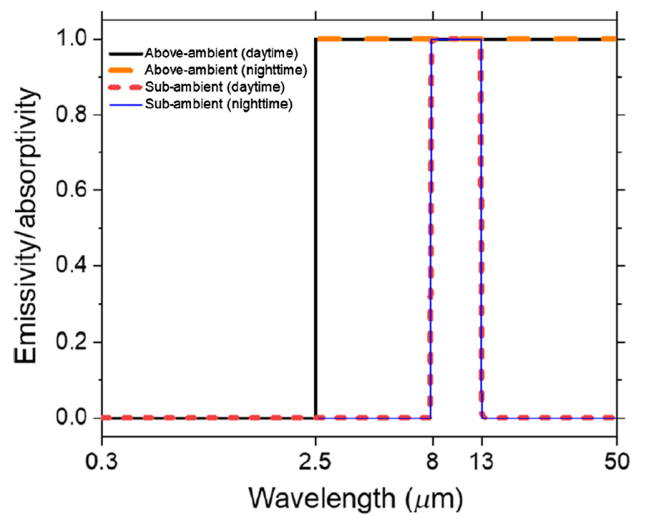

Fig. 6 The emissivity and absorptivity spectrum for various cooling scenarios, daytime aboveambient (solid black line), daytime subambient (red dashed line), nighttime above-ambient (orange dashed line), and nighttime subambient (solid blue line), respectively (reprinted from Zhao et al., ${ }^{6}$ with permission from AIP). 
Table 1 Summary of the recent development in ODR design.

\begin{tabular}{|c|c|c|c|c|c|}
\hline Structure & Materials & $\begin{array}{l}\text { ODR range } \\
\qquad(\mu \mathrm{m})\end{array}$ & $\begin{array}{l}\text { ODR width } \\
\qquad(\mu \mathrm{m})\end{array}$ & Year & References \\
\hline Planner & Polystyrene/tellurium & 10 to 15 & 5 & 1998 & Fink et al. ${ }^{80}$ \\
\hline Planner & $\mathrm{SiO}_{2}$ and $\mathrm{GaSb}$ & 1.48 to 1.68 & 0.20 & 2014 & Joseph et al ${ }^{85}$ \\
\hline Planner & $\begin{array}{l}\mathrm{KTP} / \mathrm{PBS} \text { as birefringent } \\
\text { and } \mathrm{SiO}_{2} \text { as dielectric }\end{array}$ & 3.155 to 4.202 & 1.047 & 2012 & Upadhyay et al. ${ }^{86}$ \\
\hline Graded & & 3.188 to 4.655 & 1.467 & 2018 & Kumar et al. ${ }^{87}$ \\
\hline $\begin{array}{l}\text { Decreasing width } \\
\text { multilayer }\end{array}$ & $\mathrm{CsBr} / \mathrm{Te}$ & 12 to 20 & 08 & 2017 & del Barco et al. ${ }^{137}$ \\
\hline Multistacking & $\mathrm{SiO}_{2}$ and $\mathrm{Si}_{3} \mathrm{~N}_{4}$ & 0.3 to 2.4 & 2.1 (TE only) & 2019 & Ratra et al. ${ }^{138}$ \\
\hline Multistacking & $\mathrm{SiO}_{2}$ and $\mathrm{Si}_{3} \mathrm{~N}_{4}$ & 0.3 to 2.3 & 2.0 & 2020 & Ratra et al. ${ }^{139}$ \\
\hline Planner & Porous silicon & 1.0 to 2.0 & 2.0 & 2020 & Castillo et al. ${ }^{140}$ \\
\hline
\end{tabular}

wavelength. ${ }^{79}$ Different types of $1 \mathrm{D}-\mathrm{PhC}$ structures have already been investigated to design ODRs.

Fink et al. ${ }^{80}$ and Winn et al. ${ }^{81}$ presented a 1D-PhC-based dielectric structure with nine layers of an alternating stack of polystyrene/tellurium materials and reported an ODR over the wavelength range from 10 to $15 \mu \mathrm{m}$. The ODR bandgap can also be enlarged by considering heterojunction photonic multilayer structures. ${ }^{82-84}$ Joseph et al. ${ }^{85}$ proposed a dispersive $1 \mathrm{D}-\mathrm{PhC}$ structure made of $\mathrm{SiO}_{2}$ (as low-index medium) and $\mathrm{GaSb}$ (as high index medium) and reported an ODR in 1480- to 1680-nm wavelength range. The combination of dielectric and birefringent material has also been explored for designing of ODR structures. Upadhyay et al.$^{86}$ used potassium titanyl phosphate (KTP)/lead sulfide (PBS) as birefringent materials and $\mathrm{SiO}_{2}$ as a dielectric material and reported an ODR over the wavelength range of 3.155 to $4.202 \mu \mathrm{m}$. The ODR bandgap was further enhanced by considering graded structures of same material and reported an ODR over a wavelength range of 3.188 to $4.655 \mu \mathrm{m} .^{87}$

Various natural species (i.e., Papilio palinurus butterfly, silvery fish, etc.) have also been studied to understand their ODR phenomena. Jordan et al. ${ }^{88}$ and later Zhao et al. ${ }^{89}$ studied and reported a nonpolarizing optical mechanism found in the broadband guanine-cytoplasm "silver" multilayer reflectors of three species of fish. The silvery reflections from the fish are because of the multilayer stacks of guanine crystals and index cytoplasm. ${ }^{90,91}$ These work as high and low refractive index media, respectively. Similarly, Han et al. ${ }^{23}$ studied the Papilio palinurus butterfly structure and theoretically and experimentally reported the findings of novel omnidirectional reflective self-stable properties. Furthermore, based on this study, the authors successfully designed and fabricated a $\mathrm{SiO}_{2}$-material-based bioinspired color reflector using a simple biotemplate method. Although, the reported papers clearly demonstrate capability of dielectricbased 1D-PhC structure to design ODR but width of reflection spectrum is still a tricky task to cover complete solar spectrum. Table 1 summarizes the recent work done on designing ODRs.

\subsection{Radiative Coolers Based on 1D Nanophotonic Structure}

Rephaeli et al. ${ }^{92}$ proposed a metal-dielectric-based multilayer structure with three sets of five bilayers of $\mathrm{MgF}_{2}$ and $\mathrm{TiO}_{2}$ materials as shown in Fig. 7(a). The proposed structure is embedded between two layers of $2 \mathrm{D}$ photonic crystal (2D-PhC) made of quartz and $\mathrm{SiC}$ and shows the omnidirectional reflectivity for complete solar spectrum. The proposed structure shows its capability of achieving a net cooling power of around $100 \mathrm{~W} / \mathrm{m}^{2}$ at environmental temperature. Similarly, Raman et al. ${ }^{93}$ designed a structure with planar photonic device consisting of seven alternating layers of hafnium oxide $\left(\mathrm{HfO}_{2}\right)$ and $\mathrm{SiO}_{2}$ (having different layer thicknesses) on a silver-coated silicon substrate as shown in Fig. 7(b). The bottom $\mathrm{SiO}_{2} / \mathrm{HfO}_{2}$-based thinner structure is responsible for solar reflection, while the top thicker structure is designed for thermal 


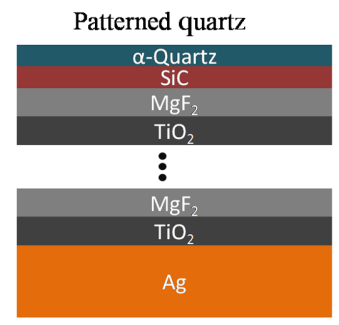

(a)

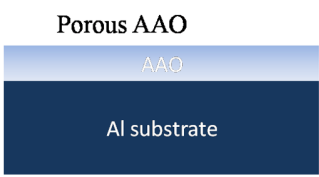

(d)

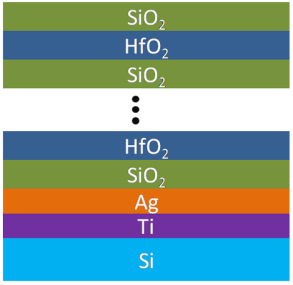

(b)

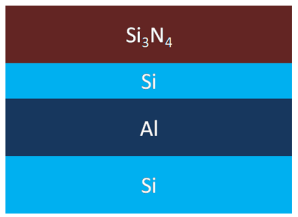

(e)

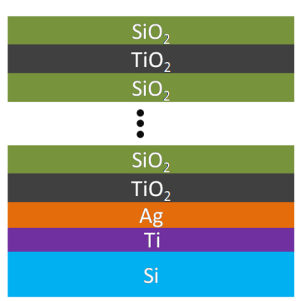

(c)

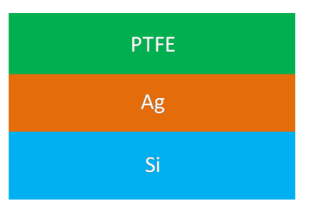

(f)

Fig. 7 Schematics of various 1D nanophotonic structures used for radiative cooling. Each design as a different layered structure: (a) $\mathrm{MgF}_{2}-\mathrm{TiO}_{2}$-based structure, ${ }^{92}$ (b) $\mathrm{HfO}_{2}-\mathrm{SiO}_{2}$ multilayer, ${ }^{93}$ (c) modified structure by replacing $\mathrm{HfO}_{2}$ with $\mathrm{TiO}_{2},{ }^{95}$ (d) porous AAO membranes-based design, ${ }^{96}$ (e) $\mathrm{Si}_{3} \mathrm{~N}_{4}-\mathrm{Si}-\mathrm{Al}$-based structure, ${ }^{97}$ and (f) PTFE and Ag on Si substrate. ${ }^{104}$

radiation. The authors experimentally reported a $97 \%$ reflection of the complete solar spectrum and an average emissivity of about 0.65 in the transparency window. They experimentally demonstrated a temperature reduction of $4.9^{\circ} \mathrm{C}$ below the ambient under direct sunlight. This structure has been widely studied by many researchers. Different materials are considered for further improvement in the net cooling power. Kecebas et al. ${ }^{94}$ replaced $\mathrm{HfO}_{2}$ with $\mathrm{TiO}_{2}$ material along with a top $\mathrm{Al}_{2} \mathrm{O}_{3}$ layer. Jeong et al. ${ }^{95}$ further optimized this $\mathrm{TiO}_{2}-\mathrm{SiO}_{2}$-based daytime radiative cooler structure with an average emissivity of 0.84 in the sky window along with $94 \%$ reflectivity of incident solar energy as shown in Fig. 7(c). The authors estimated the net cooling power around $136.3 \mathrm{~W} / \mathrm{m}^{2}$. This shows $90 \mathrm{~W} / \mathrm{m}^{2}$ improvement in cooling power compared to that of the $\mathrm{HfO}_{2} / \mathrm{SiO}_{2}$ material-based photonic radiative cooler structure. The authors successfully demonstrated a reduction of $7.2^{\circ} \mathrm{C}$ in temperature along with a net cooling power of $14.3 \mathrm{~W} / \mathrm{m}^{2}$ under direct sunlight using solar shading.

Fu et al. ${ }^{96}$ demonstrated a daytime passive radiative cooler composed of porous anodic aluminum oxide (AAO) membranes as shown in Fig. 7(d). The porosity (or air doping) and AAO thickness were optimized to get the desired results. The designed structure is capable of providing a power density of $64 \mathrm{~W} / \mathrm{m}^{2}$ at environmental temperature (at humidity of $\sim 70 \%$ ) under direct sunlight. The authors experimentally showed the structure cooling by reducing temperature by $2.6^{\circ} \mathrm{C}$ below the ambient air under direct sunlight. In another report, Chen et al. ${ }^{97}$ used silicon nitride $\left(\mathrm{Si}_{3} \mathrm{~N}_{4}\right)$, silicon ( $\left.\mathrm{Si}\right)$, and aluminum $(\mathrm{Al})$ to design a multilayer structure [as shown in Fig. 7(e)] that works as an ideal selective thermal emitter for sky window. The authors reported an average $42^{\circ} \mathrm{C}$ temperature reduction below ambient.

Furthermore, calcium fluoride $\left(\mathrm{CaF}_{2}\right)$ and germanium $(\mathrm{Ge})$-based material systems are also explored and used by Huang et al. ${ }^{98}$ Authors designed an "invisible" radiative cooling structure by considering a nichrome metal film on which seven alternating layers of $\mathrm{CaF}_{2} / \mathrm{Ge}$ were deposited. The structural parameters were optimized to get the desired results. To reduce the cost, polymer materials are also explored for this application. Polyvinyl fluoride, polyvinyl chloride, polydimethyl-siloxane (PDMS), polyethylene terephthalate, and polymethylpentene (TPX) are commonly used polymer materials, which are widely used for radiative cooling applications. ${ }^{99-102}$ These materials are beneficial as they offer very strong IR emission and possess the capability of large-scale production along with the low cost.

However, polymer material-based radiators have various concerns that are discussed in detail in later sections. Gentle et al. ${ }^{103}$ used polymer materials to demonstrate reduction in temperature below ambient. The structure was designed by considering birefringent polymer materials arranged in stacked-like structure. Thus, creating a polymer-based reflector for the visible 
spectrum. To cover the complete solar spectrum, a silver metallic layer was deposited at the bottom side, which is used to reflect the IR wavelength range of the spectrum. Based on the structures, the authors successfully demonstrated cooling of $2^{\circ} \mathrm{C}$ below the environmental temperature under the direct sunlight with the irradiation of about $1060 \mathrm{Wm}^{-2}$. In 2017, a polymercoated fused silica mirror was also proposed. The designed structure exhibits the characteristic of a near-ideal blackbody as well as near-ideal reflector in the MIR and in the solar spectrum, respectively. The authors demonstrated the radiative cooling below ambient air temperature during daytime $\left(8.2^{\circ} \mathrm{C}\right)$ and during nighttime $\left(8.4^{\circ} \mathrm{C}\right) .{ }^{26}$ Recently, Yang et al. ${ }^{104}$ used polytetrafluoroethylene (PTFE) and Ag film on Si substrate [as shown in Fig. 7(f)] and successfully demonstrated radiative cooling to $11^{\circ} \mathrm{C}$ below the environmental temperature.

The reported results demonstrated well the possibilities of 1D nanophotonic structures to control light in the multiple photonic bands (sky window and solar spectrum) that are extremely desirable for daytime passive radiative cooling applications. However, designing an ODR that can cover complete solar spectrum [ultraviolet (UV) to IR] using simple 1D photonic structures is difficult. Furthermore, the application of metal as back-reflector also affects the reflection of UV spectrum. This is because of the metallic absorption coefficient in the UV wavelength region. ${ }^{105}$ Working toward this goal, Yao et al. ${ }^{106}$ proposed a dual-band selective emitter with multinanolayers. Authors used a tandem $\mathrm{SiO}_{2} / \mathrm{Si}_{3} \mathrm{~N}_{4}$ PhC-based structure with different PBGs ( $\mathrm{PhC} 1$ and $\mathrm{PhC} 2)$, which are stacked over a silver metallic reflector. The proposed design (as shown in Fig. 8) shows its capability of radiative cooling to $11^{\circ} \mathrm{C}$ below the environmental temperature under direct sunlight.

The average emissivity of the design can also be improved using colored radiative coolers. Compared to traditional radiative cooling structures, this technique leads to increase in overall emissivity within the sky window and leads to reduction in the cooling loss. Recently, Sheng et al. ${ }^{107}$ designed a colored radiative cooler structure based on optical Tamm resonance. The proposed structure possesses the capabilities to produce high-performance cooling and can retain high-purity subtractive primary colors (CMY). The authors used the Tamm structure, composed of a $\mathrm{DBR}\left(\mathrm{MgF}_{2} / \mathrm{SiC}\right)$ on top of a silver film, and successfully presented a temperature reduction of $5^{\circ} \mathrm{C}$ to $6^{\circ} \mathrm{C}$. Table 2 summarizes the recent work done on designing radiative cooler based on 1D nanophotonic structure.

\subsection{Radiative Coolers Based on 2D Nanophotonic Structures}

Furthermore, 2D structures are also explored for designing daytime radiative coolers. Nanophotonic structures with structural dimensions around wavelength exhibit distinct radiation properties. ${ }^{108,109}$ Microresonator structures are excellent candidates to reflect or absorb a given wavelength. ${ }^{110-112}$ A number of nanophotonic structures, such as conical pillar arrays, dielectric resonator metasurfaces, metal-dielectric-metal resonators, and multilayer pyramidal nanostructures, are explored to design daytime passive radiative coolers. These structures possess the capability to tailor the spectral selectivity of the surfaces. Initially, Rephaeli et al. ${ }^{92}$ explored the possibility of daytime radiative cooling using a nanophotonic structure and successfully demonstrated a temperature reduction of almost $4.9^{\circ} \mathrm{C}$ below the ambient.

A general strategy for achieving color-preserving radiative cooling using an $\alpha$-quartz nanostructure has also been proposed. ${ }^{113}$ The structure comprises an array of $\alpha$-quartz bars

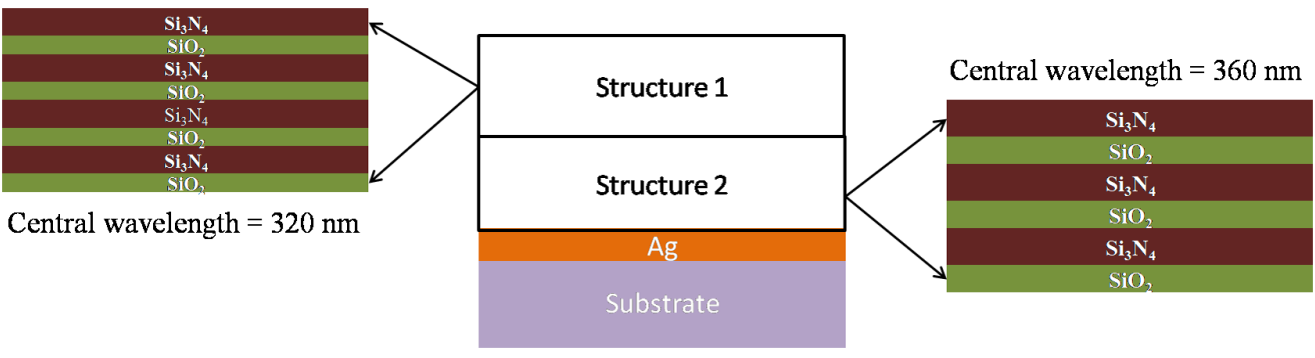

Fig. 8 Schematics of the proposed tandem PhC stack-based radiative cooling design. ${ }^{106}$ 
Table 2 Summary of the recent development in 1D nanophotonic structures.

\begin{tabular}{|c|c|c|c|c|}
\hline Materials & $\begin{array}{l}\text { Cooling power } \\
\qquad\left(\mathrm{W} / \mathrm{m}^{2}\right)\end{array}$ & $\begin{array}{l}\text { Temperature } \\
\text { reduction }\left({ }^{\circ} \mathrm{C}\right)\end{array}$ & Year & References \\
\hline $\mathrm{SiO}_{2}-\mathrm{TiO}_{2}$-based structure & $\sim 14.3$ & 7.2 & 2020 & Jeong et al. ${ }^{95}$ \\
\hline Porous AAO membranes on aluminum substrate & $\sim 64$ & 2.6 & 2019 & Fu et al. ${ }^{96}$ \\
\hline PDMS film on a reflective metal substrate & - & 3.2 & 2019 & Zho et al. ${ }^{101}$ \\
\hline $\begin{array}{l}\text { A } 150-n m \text {-thick silver }(\mathrm{Ag}) \text { and } 50 \text {-nm-thick } \mathrm{SiO}_{2} \\
\text { on a } 500-\mu \mathrm{m} \text {-thick } \mathrm{SiO}_{2} \text { substrate }\end{array}$ & - & 5.9 & 2019 & Zhao et al. ${ }^{141}$ \\
\hline Tandem $\mathrm{SiO}_{2} / \mathrm{Si}_{3} \mathrm{~N}_{4}$ & - & 11 & 2019 & Zho et al. ${ }^{101}$ \\
\hline $\mathrm{MgF}_{2} / \mathrm{SiC}$ multilayer on top of a $\mathrm{Ag}$ film & - & 5 to 6 & 2019 & Sheng et al. ${ }^{107}$ \\
\hline $\begin{array}{l}\text { Structure made by ultrawhite glass-plated } \mathrm{Ag} \\
\text { Zinc phosphate sodium }\left(\mathrm{NaZnPO}_{4}\right) \text { particles on } \\
\text { aluminum (Al) substrate }\end{array}$ & - & $\begin{array}{l}2.5 \\
1.5\end{array}$ & $\begin{array}{l}2019 \\
2019\end{array}$ & Ao et al. ${ }^{142}$ \\
\hline (PTFE) and Ag film on Si substrate & - & 11 & 2017 & Yang et al. ${ }^{104}$ \\
\hline $\begin{array}{l}\text { Two layers of acrylic resin embedded with } \mathrm{TiO}_{2} \\
\text { and carbon black particles }\end{array}$ & 100 & 6 & 2017 & Huang et al. ${ }^{76}$ \\
\hline Stack of $\mathrm{PDMS} / \mathrm{SiO}_{2} / \mathrm{Ag}$ & 127 & 8.2 & 2017 & Kou et al. ${ }^{26}$ \\
\hline
\end{tabular}

placed on top of another arrayed structure made of silicon bars [as shown in Fig. 9(a)]. The colorpreserving radiative cooling strategy can also be implemented using a multilayer photonic radiator. ${ }^{114} \mathrm{Zhu}$ et al. ${ }^{115}$ used a $\mathrm{SiO}_{2}$-based $\mathrm{PhC}$ absorber for radiative cooling applications. The oxide material is considered because of its strong phonon-polariton resonance properties in the sky window. The structure is designed on a double-sided polished fused silica substrate, in which air holes of depth $10 \mu \mathrm{m}$ are etched. The air holes are arranged in a square lattice of periodicity (lattice constant) $6 \mu \mathrm{m}$. The structure shows its capability by reducing the temperature by $13^{\circ} \mathrm{C}$ from ambient under the direct sunlight.

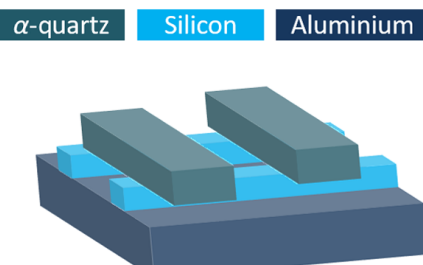

(a)

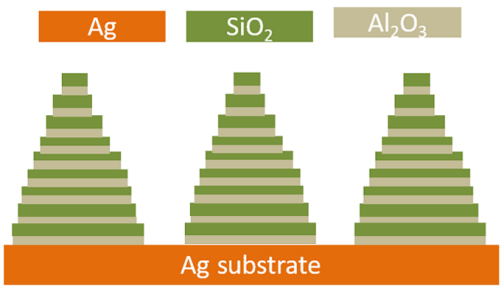

(c)

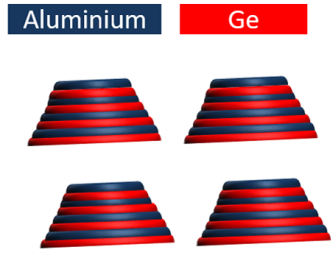

(b)

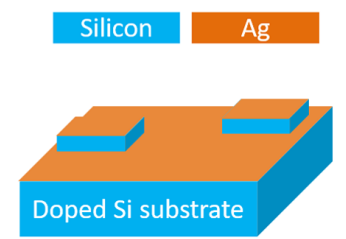

(d)

Fig. 9 Schematic of various used approaches. (a) Color-preserving radiative cooling device with $4 \alpha$-quartz bar array on top of the original structure for strong thermal emission, ${ }^{113}$ (b) Thermal emitter with multilayer conical metamaterial pillar arrays made of $\mathrm{Al}$ and $\mathrm{Ge}^{116}$ (c) Multilayer all-dielectric micropyramid structure, ${ }^{117}$ and (d) metal-loaded dielectric resonator structure. ${ }^{119}$ 
Hossain et al. ${ }^{116}$ designed a thermal emitter by introducing a conical metamaterial array made of $\mathrm{Al}$ and $\mathrm{Ge}$ material and arranged in a pillar-type structure as shown in Fig. 9(b). This is the first research carried out on antenna-type structures. The aluminum and germanium possess the thickness of around 30 and $110 \mathrm{~nm}$, respectively. The proposed structure exhibits a radiative cooling power of $116.6 \mathrm{~W} / \mathrm{m}^{2}$ at ambient temperature. Working further on the structure, Wu et al. ${ }^{117}$ reported a dielectric material-based micropyramid structure as shown in Fig. 9(c). The proposed design theoretically retains the best capability of radiative cooling effect of $47^{\circ} \mathrm{C}$ below the ambient but wide acceptability is still a concern due to its complex fabrication. Recently, Cho et al. ${ }^{118}$ reported an antenna design using tungsten (W) material. The authors designed a cone-type structure and successfully fabricated the structure using laser-interferometric lithography. They demonstrated that emission improves along with the increase of aspect ratio of cone. A simpler metalloaded dielectric resonator structure is designed by Zou et al. ${ }^{119}$ The designed surface consists of phosphorus-doped n-type silicon and silver layer as shown in Fig. 9(d). The proposed structure is advantageous in terms of integration possibility with various silicon-based platforms.

Inspired by Morpho didius butterfly wings' structure, Didari et al. ${ }^{120}$ presented a designer metamaterial system for radiative cooling applications. This nanophotonic structure consists of $\mathrm{SiC}$ material-based tree structures as shown in Fig. 10(b). The actual internal structure is schematically represented in Fig. 10(a). To enhance the spectral emissivity, the authors further optimized the structural dimensions and complete structure placed in close proximity of a thin film in a vacuum separated by nanoscale gaps as shown in Figs. 10(c) and 10(d). This modification in design improves the near-field radiative transfer within the sky window. Another studied on white beetles Goliathus has also been carried out recently. ${ }^{121}$ It possesses the unique structure comprising an exterior shell and a unique interior of packed hollow cylinders as shown in Fig. 10(e). This structure not only contributes to a lower equilibrium temperature under direct sunlight but also enhances the broadband omnidirectional reflection of solar spectrum. This is because of the thin-film interference, Mie resonance, and total internal reflection. Furthermore, the structure is also highly emissive in the MIR range. Combining all these effects, it possesses the capability to reduce their temperature approximated around $\sim 7.8^{\circ} \mathrm{C}$ in air. Table 3 summarizes the recent work done on designing radiative cooler based on $2 \mathrm{D}$ nanophotonic structure.

\section{Future Perspective and Challenges}

Development of nanophotonic devices-based passive radiator structures is a hot research topic for radiative cooling, where incorporation of photonic technology is an added advantage due to

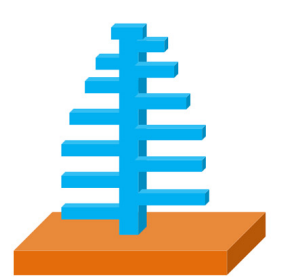

(a)

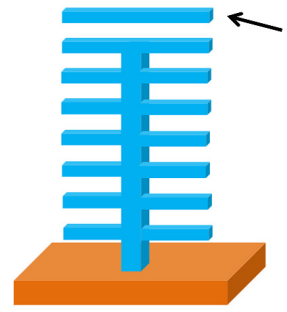

(c)

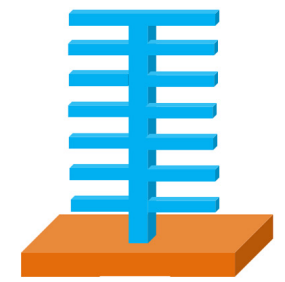

(b)

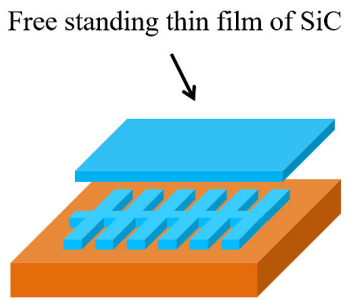

(d)

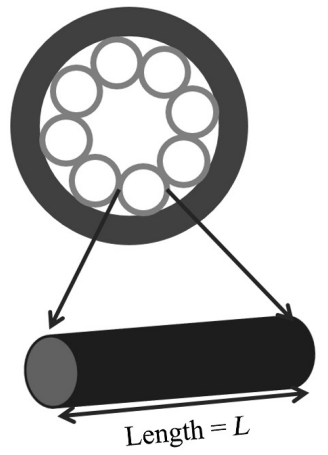

(e)

Fig. 10 Schematic illustration of (a) internal structure of Morpho didius butterfly wings', (b) proposed simplified structure, (c) and (d) modified proposed structures, ${ }^{120}$ and (e) interior cylindrical structure of white beetles Goliathus. ${ }^{121}$ 
Table 3 Summary of the recent development in 2D nanophotonic structure.

\begin{tabular}{|c|c|c|c|c|}
\hline References & $\begin{array}{l}\text { Cooling power } \\
\left(\mathrm{W} / \mathrm{m}^{2}\right)\end{array}$ & $\begin{array}{l}\text { Temperature } \\
\text { reduction }\left({ }^{\circ} \mathrm{C}\right)\end{array}$ & Year & Structure \\
\hline Zhang et al. ${ }^{143}$ & 90.8 & 5.1 & 2020 & $\begin{array}{l}\text { Photonic film comprising a micropyramid- } \\
\text { arrayed polymer matrix with random } \\
\text { ceramic particles }\end{array}$ \\
\hline Yang et al. ${ }^{144}$ & 60 & 5 & 2019 & $\begin{array}{l}\text { 1-mm-thick lithium fluoride crystal coated } \\
\text { with silver backing }\end{array}$ \\
\hline Wu et al. ${ }^{117}$ & 122 & $\begin{array}{l}47^{\circ} \mathrm{C} \text { below } \\
\text { the ambient } \\
\text { temperature }\end{array}$ & 2018 & $\begin{array}{l}\text { Two-dimensional pyramidal nanostructure } \\
\text { of the radiative cooler is composed of } \\
\text { alternating aluminum oxide }\left(\mathrm{Al}_{2} \mathrm{O}_{3}\right) \text { and } \\
\text { silica }\left(\mathrm{SiO}_{2}\right) \text { multilayer thin films and a } \\
\text { bottom silver layer }\end{array}$ \\
\hline Atiganyanun et al. ${ }^{145}$ & - & 4 & 2018 & $\begin{array}{l}\text { Randomly packed low-index } \mathrm{SiO}_{2} \\
\text { microspheres }\end{array}$ \\
\hline Mandal et al. ${ }^{146}$ & 96 & 6 & 2018 & Porous $\mathrm{P}(\mathrm{VdF}-\mathrm{HFP}) \mathrm{HP}$ coating \\
\hline Bao et al. ${ }^{147}$ & - & 5 & 2017 & $\begin{array}{l}\text { Two layers of } \mathrm{TiO}_{2}, \mathrm{SiO}_{2} \text {, and } \mathrm{SiC} \\
\text { nanoparticles }\end{array}$ \\
\hline Zhai et al. ${ }^{148}$ & 93 & - & 2017 & $\begin{array}{l}\text { Micrometer-sized } \mathrm{SiO}_{2} \text { spheres randomly } \\
\text { distributed in a matrix material of TPX }\end{array}$ \\
\hline
\end{tabular}

its unique capability to tailor the spectral properties of the radiator for designing an efficient daytime passive radiative cooling device. This has promoted the development of devices for subambient radiative cooling in recent years. Early research demonstrated that photonic technology-based structures can be used for these applications by thoroughly optimizing their properties to achieve better spectral emissivity for sky window while using a back-reflector for solar spectrum. Most of the reported work focuses on efficiency optimization of the device. However, this can also be designed to work selectively depending on environmental variation. For example, passive radiative cooling may not be required during winter and nighttime. Thereby, selfadapting radiators have desired those work according to different ambient temperatures. ${ }^{122-124}$ Thus, it becomes desirable to adopt a material whose properties change according to environmental conditions. Phase change materials seem to be best materials for these types of designs. ${ }^{125,126}$ The phase change materials convert from one phase to other by heating them up to their transition temperature. ${ }^{127,128}$ Various phase change materials, such as $\mathrm{VO}_{2}$, $\mathrm{Ge}_{2} \mathrm{Sb}_{2} \mathrm{Te}_{2}$, and modified graphene oxide, are explored in literature. ${ }^{122,129,130}$ Working toward this direction, Ono et al. ${ }^{122}$ designed a photonic structure to realize self-adaptive radiative cooling. The authors designed a spectrally selective filter made of 11 layers of $\mathrm{Ge} / \mathrm{MgF}_{2}$ on the top of a three-layer $\mathrm{VO}_{2} / \mathrm{MgF}_{2} / \mathrm{W}$ structure as shown in Fig. 11(a). Here, $\mathrm{VO}_{2}$ works as the phase change material. Similarly, Wu et al. ${ }^{123}$ also proposed a structure with $\mathrm{VO}_{2}$ [as phase change material shown in Fig. 11(d)] stacked with $\mathrm{SiO}_{2}$ and successfully demonstrated the change in cooling power by phase changing of $\mathrm{VO}_{2}$.

Furthermore, there are a number of additional challenges that need to be overcome to design an efficient radiator. The fabrication procedure of $2 \mathrm{D}$ and $3 \mathrm{D}$ photonic radiator structures is challenging that constraint them to become commercial application. ${ }^{131,132}$ Laser-interferometric lithography and femtosecond laser cross-linking techniques are explored to fabricate these complex structure. ${ }^{18,133}$ The techniques utilize a laser beam that is incident of a precursor material. The incident laser beam needs an effective control and guiding mechanism to generate such complex geometries. The femtosecond laser technique can also be used to create nanoholes array in aluminum film. ${ }^{134}$ Similarly, the 1D nanophotonic structures seem to be better as their fabrication is quite simpler than alternate $2 \mathrm{D}$ and $3 \mathrm{D}$ nanophotonic structures. Additionally, largescale production is also difficult to achieve at present. Therefore, photonic devices-based passive radiators are still in the early development stage and restricted to laboratory research and exploration. Polymer-based radiative cooling structures are likely to be the future devices due to their 


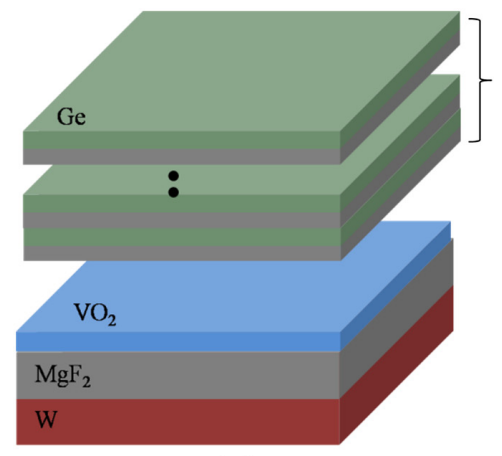

(a)
Total 11 layers

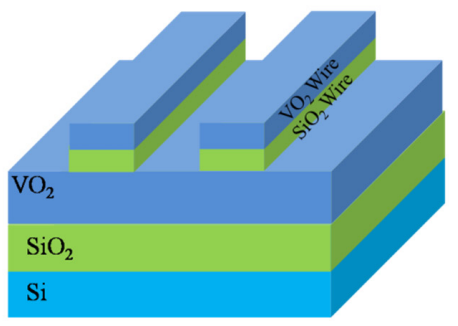

(b)

Fig. 11 Schematic of proposed switchable radiative cooling devices made of phase change material. (a) A spectrally selective filter made of 11 layers of $\mathrm{Ge} / \mathrm{MgF}_{2}$ on the top of a $\mathrm{VO}_{2} /$ $\mathrm{MgF}_{2} / \mathrm{W}$ structure ${ }^{122}$ and (b) $\mathrm{VO}_{2}$ stacked with $\mathrm{SiO}_{2}$ on silicon substrate. ${ }^{123}$

significant advantage in terms of ease of manufacturability and cost. ${ }^{135}$ However, reliability and long-term stability are the main critical challenges that will always be there. Polymer degradation with time affects their long-term high solar reflectivity. The same is true for the metal, where moisture may lead to formation of metal oxide. Thereby, exploration of a new and effective material system is always required for functionality and efficiency enhancement of the radiative cooler structures.

\section{Conclusion}

In this review, a thorough study in the field of daytime passive radiative cooling has been carried out, which focuses on recent progress made in the field. Reported works and their corresponding results demonstrate that photonic nanostructures play a significant role in the efficiency improvement of these devices. Furthermore, different nanophotonic structures, their radiative properties, and corresponding design mechanisms are discussed in details. Nature-inspired nanophotonic structures seem to have an edge for radiative cooling. However, their practical implementation is still in process. Furthermore, in the future, the key technologies of biomimetic daytime radiator will be the development, device integration, and explorations of new designs and methods, which will open a new way to design highly efficient passive daytime radiative coolers.

\section{Acknowledgments}

Authors acknowledge all members of ECE Department at JIIT for their help and support. Authors declare no conflicts of interest.

\section{References}

1. E. D. Cian et al., "Global energy consumption in a warming climate," Environ. Resource Econ. 72(2), 365-410 (2019).

2. O. Meangbua et al., "Factors influencing energy requirements and $\mathrm{CO}_{2}$ emissions of households in Thailand: a panel data analysis," Energy Policy 129, 521-531 (2019).

3. M. I. Ahmad et al., "Introduction: overview of buildings and passive cooling technique," in Nocturnal Cooling Technology for Building Applications, Springer Briefs in Applied Sciences and Technology, Springer, Singapore (2019).

4. D. Sato et al., "Review of photovoltaic module cooling methods and performance evaluation of the radiative cooling method," Renew. Sustain. Energy Rev. 104, 151-166 (2019).

5. M. Santamouris, Cooling Energy Solutions for Buildings and Cities, University of New South Wales, Sydney (2019). 
6. D. Zhao et al., "Radiative sky cooling: fundamental principles, materials, and applications," Appl. Phys. Rev. 6, 021306 (2019).

7. C. D. Reid et al., "Measurement of spectral emissivity from $2 \mu \mathrm{m}$ to $15 \mu \mathrm{m}$," JOSA 49, 78-82 (1959).

8. H. Miyazaki et al., "Fabrication of radiative cooling devices using $\mathrm{Si}_{2} \mathrm{~N}_{2} \mathrm{O}$ nano-particles," J. Ceram. Soc. Jpn. 124, 1185-1187 (2016).

9. H. Miyazaki et al., "Fabrication of radiative cooling materials based on $\mathrm{Si}_{2} \mathrm{~N}_{2} \mathrm{O}$ particles by the nitridation of mixtures of silicon and silicon dioxide powders," J. Ceram. Soc. Jpn. 121, 242-245 (2013).

10. B. Zho et al., "Performance analysis of a hybrid system combining photovoltaic and night time radiative cooling," Appl. Energy 252, 113432 (2019).

11. C. G. C. Granqvist et al., "Radiative cooling to low temperatures: general considerations and application to selectively emitting SiO films," J. Appl. Phys. 52, 4205 (1981).

12. R. Craig, The Upper Atmosphere: Meteorology And Physics, Academic Press, New York (1965).

13. A. Rephaeli et al., "Ultra broadband photonic structures to achieve high-performance day-time radiative cooling," Nano Lett. 13, 1457-1461 (2013).

14. D. Liu et al., "A thermally stable cooler for efficient passive radiative cooling throughout the day," Opt. Mater. 92, 330-334 (2019).

15. T. M. J. Nilsson et al., "A solar reflecting material for radiative cooling applications: $\mathrm{ZnS}$ pigmented polyethylene," Sol. Energy Mater. Sol. Cells 28, 175-193 (1992).

16. T. M. J. Nilsson et al., "Radiative cooling during the day: simulations and experiments on pigmented polyethylene cover foils," Sol. Energy Mater. Sol. Cells 37, 93-118 (1995).

17. T. Mouhib et al., "Stainless steel/tin/glass coating as spectrally selective material for passive radiative cooling applications," Opt. Mater. 31, 673-677 (2009).

18. J. Peoples et al., "A strategy of hierarchical particle sizes in nanoparticle composite for enhancing solar reflection," Int. J. Heat Mass Transfer 131, 487-494 (2019).

19. H. Yuan et al., "Effective, angle-independent radiative cooler based on one-dimensional photonic crystal," Opt. Express 26(21), 27885-27893 (2018).

20. W. Liu et al., "Multilayer thin-film meta-surface thermal emitter for radiation cooling in high-temperature devices," in Int. Photonics and Optoelectron. Meeting, OSA Technical Digest, Paper OT4A.70, Optical Society of America (2018).

21. Y. Zhu et al., "Thin films of high reflectivity for efficient radiative cooling," Proc. SPIE 10964, 109643U (2018).

22. N. Athanasopoulos et al., "Programmable thermal emissivity structures based on bioinspired self-shape materials," Sci. Rep. 5, 17682 (2015).

23. Z. Han et al., "Bio-inspired omnidirectional self-stable reflectors with multi-scale hierarchical structures," ACS Appl. Mater. Interfaces 9, 29285-29294 (2017).

24. Z. Ouyang et al., "Photonic structures based on dielectric and magnetic one-dimensional photonic crystals for wide omnidirectional total reflection," JOSA-B 25(3), 297-301 (2008).

25. D. Fu, "Radiation transfer in the atmosphere: radiation, solar," in Encyclopedia of Atmospheric Science, G. R. North, J. Pyle, and F. Zhang, Eds., pp. 1-4, Elsevier, New York (2015).

26. J.-L. Kou et al., "Daytime radiative cooling using near-black infrared emitters," ACS Photonics 4(3), 626-630 (2017).

27. N. N. Shi et al., "Keeping cool: enhanced optical reflection and radiative heat dissipation in Saharan silver ants," Science 349(6245), 298-301 (2015).

28. R. H. Siddique et al., "Theoretical and experimental analysis of the structural pattern responsible for the iridescence of Morpho butterflies," Opt. Express 21, 14351-14361 (2013).

29. S. C. Cary et al., "Worms bask in extreme temperatures," Nature 391, 545-546 (1998).

30. W. J. Gehring et al., "Heat shock protein synthesis and thermo tolerance in Cataglyphis, an ant from the Sahara Desert," Proc. Natl. Acad. Sci. U. S. A. 92, 2994-2998 (1995).

31. D. D. Horikawa, "Survival of tardigrades in extreme environments: a model animal for astrobiology," in Cellular Origin, Life in Extreme Habitats and Astrobiology, Vol. 21, pp. 205-217 (2012). 
32. S. Ling et al., "Nanofibrils in nature and materials engineering," Nat. Rev. Mater. 3, 18016 (2018).

33. T. B. H. Schroeder et al., "It's not a bug, it's a feature: functional materials in insects," Adv. Mater. 30(19), 1705322 (2018).

34. $\mathrm{X}$. Wu et al., "Extreme optical properties tuned through phase substitution in a structurally optimized biological photonic polycrystal," Adv. Funct. Mater. 23(29), 3615-3620 (2013).

35. P. Vukusic et al., "Brilliant whiteness in ultrathin beetle scales," Science 315(5810), 348 (2007).

36. L. Cortese et al., "Anisotropic light transport in white beetle scales," Adv. Opt. Mater. 3(10), 1337-1341 (2015).

37. G. Smith et al., "Nanophotonics-enabled smart windows, buildings and wearables," Nanophotonics 5(1), 55-73 (2016).

38. A. Krishna et al., "Morpho butterfly-inspired spectral emissivity of metallic microstructures for radiative cooling," in 17th IEEE Intersoc. Conf. Thermal and Thermomech. Phenom. Electron. Syst., San Diego, California, pp. 78-85 (2018).

39. C. C. Tsai et al., "Butterflies regulate wing temperatures using radiative cooling," in Conf. Lasers and Electro-Opt. (2017).

40. Q. Willot et al., "Total internal reflection accounts for the bright color of the Saharan silver ant," PLoS One 11(4), e0152325 (2016).

41. N. N. Shi et al., "Nano-structured wild moth cocoon fibers as radiative cooling and waveguiding optical materials," in Conf. Lasers and Electro-Opt. (2017).

42. N. N. Shi et al., "Nano-structured fibers as a versatile photonic platform: radiative cooling and waveguiding through transverse Anderson localization," Light: Sci. Appl. 7(37), 1-9 (2018).

43. S. H. Choi et al., "Anderson light localization in biological nanostructures of native silk," Nat. Commun. 9, 452 (2018).

44. T. L. Bergman et al., Fundamentals of Heat and Mass Transfer, John Wiley \& Sons, Hoboken, New Jersey (2011).

45. Y. Liu et al., "A pragmatic bilayer selective emitter for efficient radiative cooling under direct sunlight," Materials 12(8), 1208 (2019).

46. L. Peng et al., "Design and fabrication of the ultrathin metallic film-based infrared selective radiator," Solar Energy Mater. Solar Cells 193, 7-12 (2019).

47. Y. Q. Wang et al., "Perfect electromagnetic and sound absorption via sub-wavelength holes array," Opto-Electron. Adv. 1(8), 180013 (2018).

48. J. Yang et al., "Design and fabrication of broadband ultralow reflectivity black Si surfaces by laser micro/nano processing," Light: Sci. Appl. 3(7), e185 (2014).

49. E. E. Bell et al., "Spectral radiance of sky and Terrain at wavelengths between 1 and 20 microns. II. Sky measurements," J. Opt. Soc. Am. 50(12), 1313 (1960).

50. A. W. Harrison, "Effect of atmospheric humidity on radiation cooling," Sol. Energy 26(3), 243 (1981).

51. K. Y. Kondratyev, Radiation in the Atmosphere, Academic Press, New York (1969).

52. W. Wang et al., "The radiative effect of aerosols in the Earth's atmosphere," J. Appl. Meterol. 13(5), 521-534 (1974).

53. A. V. Tikhonravov et al., "Application of the needle optimization technique to the design of optical coatings," Appl. Opt. 35, 5493-5508 (1996).

54. A. V. Tikhonravov et al., "Optical coating design approaches based on the needle optimization technique," Appl. Opt. 46, 704-710 (2007).

55. T. Boudet et al., "Thin-film designs by simulated annealing," Appl. Opt. 35, 6219-6226 (1996).

56. L. Li et al., "Jump method for optical thin film design," Opt. Express 17, 16920-16926 (2009).

57. Y. Shi et al., "Optimization of multilayer optical films with a memetic algorithm and mixed integer programming," ACS Photonics 5, 684-691 (2018).

58. C. G. Granqvist et al., "Radiative cooling to low temperatures with selectivity IR-emitting surfaces," Thin Solid Films 90, 187-190 (1982). 
59. S. Catalanotti et al., "The radiative cooling of selective surfaces," Sol. Energy 17, 83-89 (1975).

60. B. Orel et al., "Radiative cooling efficiency of white pigmented paints," Sol. Energy 50, 477-482 (1993).

61. T. S. Eriksson et al., "Surface coatings for radiative cooling applications: silicon dioxide and silicon nitride made by reactive RF sputtering," Sol. Energy Mater. 12, 319-325 (1985).

62. H. S. Dutta, A. K. Goyal, and S. Pal, "Analysis of dispersion diagram for high performance refractive index sensor based on photonic crystal waveguides," Photonics Nanostruct. Fundam. Appl. 23, 21-27 (2017).

63. J. D. Joannopoulos et al., Photonic Crystals: Molding the Flow of Light, Princeton University Press, New Jersey (2008).

64. T. Baba, "Slow light in photonic crystals," Nat. Photonics 2, 465-473 (2008).

65. A. K. Goyal et al., "Porous photonic crystal structure for sensing applications," J. Nanophotonics 12(4), 040501 (2018).

66. A. K. Goyal et al., "Performance optimization of photonic crystal resonator based sensor," Opt. Quantum Electron. 48, 431 (2016).

67. H. S. Dutta et al., "Sensitivity enhancement in photonic crystal waveguide platform for refractive index sensing applications," J. Nanophotonics 8(1), 083088 (2014).

68. Z. Li et al., "Two designs of thin film for cooling buildings based on photonic crystal," in Int. Conf. Ind. Electron. and Appl. (2015).

69. A. Ghanekar et al., "Photonic metamaterials: controlling nanoscale radiative thermal transport," in Heat Transfer-Models, Methods and Applications, K. Volkov, Ed., IntechOpen, London (2017).

70. H. Zhou et al., "Bio-inspired photonic materials: prototypes and structural effect designs for applications in solar energy manipulation," Adv. Funct. Mater. 28, 1705309 (2018).

71. K. Xu et al., "Micro optical sensors based on avalanching silicon light-emitting devices monolithically integrated on chips," Opt. Mater. Express 9, 3985-3997 (2019)

72. A. K. Goyal et al., "Porous multilayer photonic band gap structure for optical sensing," in OSA Tech. Digest, Paper Tu4A.12, Optical Society of America (2016).

73. A. K. Goyal and S. Pal, "Design analysis of Bloch surface wave based sensor for haemoglobin concentration measurement," Appl. Nanosci., accepted article (2020).

74. M. Hossain et al., "Radiative cooling: principles, progress, and potentials," Adv. Sci. 3, 1500360 (2016).

75. Y. Zahu et al., "A multilayer emitter close to ideal solar reflectance for efficient daytime radiative cooling," Polymers 11(7), 1203 (2019).

76. Z. Huang et al., "Nano-particle embedded double-layer coating for daytime radiative cooling," Int. J. Heat Mass Transf. 104, 890-896 (2017).

77. L. Zhu et al., "Radiative cooling of solar cells," Optica 1, 32-38 (2014).

78. E. Yablonovitch, "Inhibited spontaneous emission in solid-state physics and electronics," Phys. Rev. 58(20), 2059-2062 (1987).

79. A. K. Goyal et al., "Recent advances and progress in photonic crystal-based gas sensors," J. Phys. D: Appl. Phys. 50(20), 203001 (2017).

80. Y. Fink et al., "An omni-directional reflector," Science 282, 1679 (1998).

81. J. N. Winn et al., "Omnidirectional reflection from a one-dimensional photonic crystal," Opt. Lett. 23, 1573 (1998).

82. B. Gallas et al., "Making an omni-directional reflector," Appl. Opt. 40, 5056 (2001).

83. X. Wang et al., "Enlargement of omnidirectional total reflection frequency range in onedimensional photonic crystals by using photonic heterostructures," Appl. Phys. Lett. 80, 4291 (2002).

84. C. Zhang et al., "Enlargement of omnidirectional total reflection frequency range in onedimensional photonic crystals by using photonic hetero-structures," Appl. Phys. Lett. 87, 3174 (2000).

85. S. Joseph et al., "Omnidirectional reflector using one-dimensional dispersive photonic hetero-structure," Optik 125, 2734-2738 (2014). 
86. M. Upadhyay et al., "Infrared omnidirectional mirror based on one-dimensional birefringent-dielectric photonic crystal," PIER M 25, 211 (2012).

87. R. Kumar et al., "Study of one-dimensional nano-layered graded photonic crystal consisting of birefringent and dielectric materials," Photonics Nanostruct. - Fundam. Appl. 28, 20-31 (2018).

88. T. M. Jordan et al., "Non-polarizing broadband multilayer reflectors in fish," Nat Photonics 6(11), 759-763 (2012).

89. S. Zhao et al., "Broadband and polarization reflectors in the lookdown, Selene vomer," J. R. Soc. Interface 12, 20141390 (2015).

90. E. J. Denton et al., "Polarization of light reflected from the silvery exterior of the bleak, Alburnus alburnus," J. Mar. Biol. Assoc. U.K. 45(3), 705-709 (1965).

91. A. Adibi et al., "Secondary grating formation by readout at Bragg-null incidence," Appl. Opt. 38, 4291-4295 (1999).

92. E. Rephaeli et al., "Ultra broad band photonic structures to achieve high-performance daytime radiative cooling," Nano Lett. 13(4), 1457-1461 (2013).

93. P. Raman et al., "Passive radiative cooling below ambient air temperature under direct sunlight," Nature 515, 540 (2014).

94. M. A. Kecebas et al., "Passive radiative cooling design with broadband optical thin-film filters," J. Quant. Spectrosc. Rad. Transfer 198, 179-86 (2017).

95. S. Y. Jeong et al., "Field investigation of a photonic multi-layered $\mathrm{TiO}_{2}$ passive radiative cooler in sub-tropical climate," Renew. Energy 146, 44-55 (2020).

96. Y. Fu et al., "Daytime passive radiative cooler using porous alumina," Solar Energy Mater. Solar Cells 191, 50-54 (2019).

97. Z. Chen et al., "Radiative cooling to deep sub-freezing temperatures through a 24-h day-night cycle," Nat. Commun. 7, 13729 (2016).

98. Y. Huang et al., "Broadband metamaterial as an 'invisible' radiative cooling coat," Opt. Commun. 407, 204-207 (2018).

99. B. Bartoli et al., "Nocturnal and diurnal performances of selective radiators," Appl. Energy 3(4), 267-286 (1977).

100. B. Landro et al., "Effect of surface characteristics and atmospheric conditions on radiative heat loss to a clear sky," Int. J. Heat Mass Transfer 23(5), 613-620 (1980).

101. B. Zho et al., "General strategy of passive sub-ambient daytime radiative cooling," Solar Energy Mater. Solar Cells 199, 108-113 (2019).

102. M. Hu et al., "Field test and preliminary analysis of a combined diurnal solar heating and nocturnal radiative cooling system," Appl. Energy 179, 899-908 (2016).

103. A. R. Gentle et al., "A sub-ambient open roof surface under the mid-summer sun," Adv. Sci. 2, 1500119 (2015).

104. P. Yang et al., "A dual-layer structure with record-high solar reflectance for day time radiative cooling," Sol. Energy 169, 316-324 (2018).

105. E. D. Palik, Handbook of Optical Constants of Solids, Academic Press: Orlando, Florida (1985).

106. K. Yao et al., "Near-perfect selective photonic crystal emitter with nanoscale layers for daytime radiative cooling," ACS Appl. Nano Mater. 2(9), 5512-5519 (2019).

107. C. Sheng et al., "Colored radiative cooler under optical Tamm resonance," ACS Photonics, 6(10), 2545-2552 (2019).

108. K. Xu, "Silicon MOS optoelectronic micro-nano structure based on reverse-biased PN junction," Phys. Status Solidi A 216, 1800868 (2019).

109. W. Li et al., "Nano-photonic control of thermal radiation for energy applications [invited]," Opt. Express 26, 15995 (2018).

110. Y. Zheng et al., "Sensing and lasing applications of whispering gallery mode microresonators," Opto-Electron. Adv. 1(9), 180015 (2018).

111. A. E. Kaplan et al., "Tunable narrow band optical reflector based on indirectly coupled micro ring resonators," Opt. Express 28, 13497-13515 (2020).

112. A. K. Goyal et al., "Design and analysis of photonic crystal micro-cavity based optical sensor platform," AIP Conf. Proc. 1724, 020005 (2016). 
113. L. Zhu et al., "Color-preserving daytime radiative cooling," Appl. Phys. Lett. 103, 223902 (2013).

114. W. Li et al., "Photonic thermal management of coloured objects," Nat. Commun. 9, 4240 (2018).

115. L. Zhu et al., "Radiative cooling of solar absorbers using a visibly transparent photonic crystal thermal blackbody," Proc. Natl. Acad. Sci. U. S.A. 112, 12282-12287 (2015).

116. M. M. Hossain et al., "A metamaterial emitter for highly efficient radiative cooling," Adv. Opt. Mater. 3, 1047-1051 (2015).

117. D. Wu et al., "The design of ultra-broadband selective near-perfect absorber based on photonic structures to achieve near-ideal daytime radiative cooling," Mater. Des. 139, 104-111 (2018).

118. J. Cho et al., "Visible to near-infrared thermal radiation from nano-structured tungsten antennas," J. Opt. 20, 09LT01 (2018).

119. C. Zou et al., "Metal-loaded dielectric resonator metasurfaces for radiative cooling," Adv. Opt. Mater. 5, 1700460 (2017).

120. A. Didari et al., "A biomimicry design for nanoscale radiative cooling applications inspired by Morpho didius butterfly," Sci. Rep. 8, 16891 (2018).

121. D. Xie et al., "Broadband omnidirectional light reflection and radiative heat dissipation in white beetles Goliathus goliatus," Soft Matter. 15, 4294-4300 (2019).

122. M. Ono et al., "Self-adaptive radiative cooling based on phase change materials," Opt. Express 26, A777-A787 (2018).

123. S. R. Wu et al., "Passive temperature control based on a phase change metasurface," Sci. Rep. 8, 7684 (2018).

124. Y. Qu et al., "Tunable dual-band thermal emitter consisting of single-sized phase-changing GST nanodisks," Opt. Express 26, 4279-4287 (2018).

125. A. Gentle et al., "Design, control, and characterization of switchable radiative cooling," Proc. SPIE 10759, 107590L (2018).

126. K. Sun et al., "Metasurface optical solar reflectors using AZO transparent conducting oxides for radiative cooling of spacecraft," ACS Photonics 5, 495-501 (2018).

127. A. J. Noel et al., "Phase change materials," Chapter 13 in Storing Energy, T. M. Letcher, Ed., pp. 249-272, Elsevier, New York (2016).

128. X. Zhilin et al., "Easy way to achieve self-adaptive cooling of passive radiative materials," ACS Appl. Mater. Interfaces 12(24), 27241-27248 (2020).

129. Y. G. Chen et al., "Hybrid phase-change plasmonic crystals for active tuning of lattice resonances," Opt. Express 21(11), 13691-13698 (2013).

130. G. Y. Cao et al., "An accurate design of graphene oxide ultrathin flat lens based on Rayleigh-Sommerfeld theory," Opto-Electron. Adv. 1, 180012 (2018).

131. A. K. Goyal et al., "Realization of large-scale photonic crystal cavity-based devices," J. Micro/Nanolithogr. MEMS MOEMS 15(3), 031608 (2016).

132. H. S. Dutta et al., "Fabrication of photonic crystal line defect waveguides by use of optical lithography and focused ion beam," in OSA Tech. Digest, Paper w4e.4 (2016).

133. D. Serien and K. Sugioka, "Fabrication of three-dimensional proteinaceous micro- and nano-structures by femtosecond laser cross-linking," Opto-Electron. Adv. 1(4), 180008 (2018).

134. H. Liu et al., "Self-organized periodic microholes array formation on aluminium surface via femtosecond laser ablation induced incubation effect," Adv. Funct. Mater. 29(42), 1903576 (2019).

135. M. Santamouris and J. Feng, "Recent progress in daytime radiative cooling: is it the air conditioner of the future?" Buildings 8, 168 (2018).

136. "Reference solar spectral irradiance: air mass 1.5," http://rredc.nrel.gov/solar/spectra/ am1.5/.

137. O. del Barco et al., "Omnidirectional high-reflectivity mirror in the 4-20 $\mu \mathrm{m}$ spectral range," J. Opt. 19, 065102 (2017).

138. K. Ratra et al., "Design and analysis of broadband reflector for passive radiative cooling," Int. Conf. Signal Process. and Commun., Noida, pp. 300-303 (2019). 
139. K. Ratra et al., "Design and analysis of omni-directional solar spectrum reflector using one-dimensional photonic crystal," J. Nanophotonics 14(2), 026005 (2020).

140. B. A. Castillo et al., "A wide band porous silicon omnidirectional mirror for the near infrared range," J. Appl. Phys. 127, 203106 (2020).

141. B. Zhao et al., "Performance evaluation of daytime radiative cooling under different clear sky conditions," Appl. Thermal Eng. 155, 660-666 (2019).

142. X. Ao et al., "Preliminary experimental study of a specular and a diffuse surface for daytime radiative cooling," Solar Energy Mater. Solar Cells 191, 290-296 (2019).

143. H. Zhang et al., "Biologically inspired flexible photonic films for efficient passive radiative cooling," Proc. Natl. Acad. Sci. U. S. A. 117(26), 14657-14666 (2020).

144. Y. Yang et al., "Bulk material based selective infrared emitter for sub-ambient daytime radiative cooling," Sol. Energy Mater. Sol. Cells 211, 110548 (2019).

145. S. Atiganyanun et al., "Effective radiative cooling by paint-format micro-sphere based photonic random media," ACS Photonics 5, 1181-1187 (2018).

146. J. Mandal et al., "Hierarchically porous polymer coatings for highly efficient passive daytime radiative cooling," Science 362, 315-319 (2018).

147. H. Bao et al., "Double-layer nano-particle based coatings for efficient terrestrial radiative cooling," Sol. Energy Mater. Sol. Cell. 168, 78-84 (2017).

148. Y. Zhai et al., "Scalable manufactured randomized glass-polymer hybrid metamaterial for daytime radiative cooling," Science 355, 1062-1066 (2017).

Biographies of the authors are not available. 